\title{
Costs associated with the administration of erythropoiesis-stimulating agents for the treatment of anemia in patients with non-dialysis-dependent chronic kidney disease: a US societal perspective
}

\author{
Marjolaine Gauthier-Loiselle, PhD; Steven N Michalopoulos, MPH; Martin Cloutier, MSc; Elizabeth Serra, MSc; \\ Rebecca Bungay, MSc; Erika Szabo, MPH, MSPharm; and Annie Guérin, MSc
}

\section{What is already known about this subject}

- Needle-based erythropoiesisstimulating agents (ESAs) are commonly used to treat anemia resulting from chronic kidney disease (CKD).

- Despite the clinical utility of ESAs, their use is associated with a considerable economic and societal burden resulting from the logistics of therapy administration for both patients and caregivers, especially for non-dialysis-dependent (NDD) patients who do not have prescheduled regular visits for dialysis during which ESAs can be administered.

- The magnitude of the administration costs that could potentially be avoided with the introduction of a new oral treatment for anemia due to CKD with comparable safety and efficacy to ESAs has not been assessed.

\section{What this study adds}

- This study estimated the direct and indirect excess costs associated with the needle-based administration of ESAs for the treatment of anemia due to NDD-CKD at $\$ 2.5$ billion in the United States in 2019 , or $\$ 5,399$ per treated patient.

- More than $90 \%$ of this excess cost was due to in-clinic ESA administration, suggesting that resources associated with ESA administration visits account for the vast majority of costs that could potentially be avoided with the introduction of novel oral medications.

- Direct health care costs and patients' work productivity loss costs, particularly those resulting from in-clinic ESA administration, were the 2 main drivers of total excess costs, respectively, accounting for $54.9 \%$ and $33.9 \%$ of total excess costs.

\section{ABSTRACT}

BACKGROUND: Erythropoiesis-stimulating agents (ESAs) are commonly used to treat anemia due to chronic kidney disease (CKD). In addition to drug acquisition costs, the administration of ESAs can include direct and indirect costs due to the needle-based route of administration (eg, time spent by health care staff administering therapy, and patients' and caregivers' time spent receiving or assisting with therapy). However, a comprehensive assessment of the costs associated with the administration of ESAs is lacking.

\section{Author affiliations}

Marjolaine Gauthier-Loiselle, PhD; Martin Cloutier, MSc; Elizabeth Serra, MSc; Rebecca Bungay, MSc; and Annie Guérin, MSc, Analysis Group, Inc., Montreal, Quebec, Canada. Steven N Michalopoulos, MPH, Otsuka Pharmaceutical Development \& Commercialization, Inc., Rockville, MD, and Erika Szabo, MPH, MSPharm, Akebia Therapeutics, Inc., Cambridge, MA.

\section{AUTHOR CORRESPONDENCE:}

Martin Cloutier, 514.394.4441;

Martin.Cloutier@analysisgroup.com

J Manag Care Spec Pharm 2021;27(12):1703-13

Copyright $\odot 2021$, Academy of Managed Care Pharmacy. All rights reserved.

OBJECTIVE: To estimate the excess costs associated with the needle-based administration of ESAs for the treatment of anemia due to non-dialysis-dependent (NDD) CKD in the United States in 2019 from a societal perspective. 
METHODS: Excess costs associated with ESA administration were estimated as the sum of annual costs that could be avoided with the introduction of an oral treatment with comparable safety and efficacy to ESAs. Cost components included direct health care costs, transportation costs, and work productivity loss costs from the perspective of both patients and caregivers (as applicable). Costs were estimated based on scientific publications, governmental agencies, and the results of a recent survey of US patients and caregivers of patients with anemia and CKD. The setting of the administration (ie, at home vs in clinic), frequency of administration, and insurance type were considered.

RESULTS: At the societal level, annual excess costs associated with ESA administration were estimated at $\$ 2.5$ billion in the United States in 2019, based on an estimated 462,005 patients with anemia and NDD-CKD treated with ESAs. Overall, $94.4 \%$ (\$2.4 billion) of these costs were incurred from in-clinic ESA administration. When stratifying costs by insurance type, Medicare-insured patients accounted for $79.4 \%$ ( $\$ 2.0$ billion) of total annual excess costs. The largest contributor to total annual excess costs was direct health care costs ( $\$ 1.4$ billion, $54.9 \%)$, followed by patient work productivity loss costs ( $\$ 846$ million, $33.9 \%$ ), caregiver work productivity loss costs (\$197 million, 7.9\%), and transportation costs (\$81 million, 3.3\%). Total annual excess costs of in-clinic administration ranged from $\$ 2,572$ per patient receiving monthly administration to $\$ 20,948$ per patient receiving thrice-weekly administration, while the total annual excess costs of at-home administration ranged from $\$ 1,123$ per patient receiving monthly administration to $\$ 2,109$ per patient receiving thrice-weekly administration. At the ESA administration level (ie, for each ESA administration), total excess costs were estimated at \$128 per in-clinic ESA administration and \$7 per at-home ESA administration, excluding monitoring costs.

CONCLUSIONS: The needle-based administration of ESAs in patients with NDD-CKD is associated with a substantial economic burden. The introduction of an oral treatment has the potential to result in important cost savings from a societal perspective.

Chronic kidney disease (CKD) is characterized by persistent abnormalities in kidney structure, function, or both, with implications for the health of the affected individual. ${ }^{1-3}$ The prevalence of CKD increases with age, ${ }^{4}$ such that the prevalence among the Medicare-insured population is estimated to be approximately $22 \%$ compared with approximate prevalence of $4 \%$ among Medicaid-insured, $1 \%$ among commercially insured, and $<1 \%$ among uninsured populations. ${ }^{5}$ Further, both the prevalence and economic burden of the disease are on the rise, ${ }^{6}$ with total Medicare spending for CKD (including end-stage renal disease) exceeding \$120 billion in 2017, up by $5.3 \%$ relative to 2016 .

Further, the economic burden of CKD, including health care resource use and costs, significantly increases with
CKD progression, largely due to the emergence of complications. ${ }^{8,9}$ Anemia is a common complication of CKD whose prevalence markedly increases with disease progression; a study by Stauffer et al reported that anemia prevalence increases from $8.4 \%$ among patients with stage 1 CKD to $53.4 \%$ among those with stage $5 \mathrm{CKD} .{ }^{10}$ Anemia due to CKD is characterized by several symptoms (eg, fatigue, weakness, shortness of breath, insomnia, depression, dizziness) that adversely affect patients' health-related quality of life (HRQOL). ${ }^{11}$

The symptoms associated with anemia and their resulting adverse impact on HRQOL can be alleviated with appropriate treatments of anemia. ${ }^{12,13}$ Treatments currently recommended for anemia due to CKD include iron administration, erythropoiesis-stimulating agents (ESAs), and red blood cell transfusion. ${ }^{14}$ ESAs, which can be administered intravenously or subcutaneously, are commonly used for the treatment of anemia due to CKD but may introduce a substantial burden. ${ }^{14}$ In addition to drug acquisition costs, the time spent by health care professionals on ESA administration significantly contributes to the total treatment costs. ${ }^{15,16}$ These direct health care costs are further compounded by indirect work productivity loss costs due to patients' time spent receiving therapy. Furthermore, caregivers' time spent accompanying patients to therapy contributes to the societal burden of ESA treatment. ${ }^{17}$ The costs associated with ESA administration may be particularly significant in the non-dialysis-dependent (NDD) population since these patients do not have prescheduled regular visits for dialysis during which ESAs can be administered. ${ }^{18}$

The direct and indirect costs associated with the needlebased administration of ESAs may be substantially reduced with the introduction of new oral treatments for anemia due to CKD. ${ }^{19,20}$ Hypoxia-inducible factor prolyl hydroxylase inhibitors (HIF-PHIs) are a novel class of oral treatments that have demonstrated promising results in treating anemia due to CKD in recent trials. ${ }^{21,22}$ However, as prior studies have not provided a comprehensive assessment of costs incurred by patients, caregivers, and payers for the needlebased administration of ESAs, ${ }^{17,23,24}$ the potential savings that might result from the introduction of oral HIF-PHIs remain largely unknown. Consistent with the approach advocated by several health technology assessment bodies, assessing costs from a societal perspective is important to reflect the perspective of decision makers, who must take into account the broader impact of health care policies on society in order to appropriately allocate resources.

The goal of this study was to estimate the excess costs associated with the needle-based administration of ESAs for the treatment of anemia due to NDD-CKD 
in the United States in 2019 from a societal perspective. This study focused on patients with NDD-CKD, since it is challenging to accurately distinguish costs specific to the administration of ESAs from those associated with dialysis in the dialysis-dependent (DD) population (eg, if ESAs are administered during a visit for dialysis).

\section{Methods}

\section{STUDY DESIGN}

Total excess costs associated with the administration of ESAs in patients with anemia due to NDD-CKD were estimated as the sum of costs expected to be avoided with an oral treatment instead of a needle-based treatment, assuming a comparable safety and efficacy profile. Inputs used to estimate the total excess costs in the United States (ie, individual costs and prevalence estimates) were obtained from the literature, including scientific publications and governmental agencies, as well as the results of a survey conducted in 2020 among US patients and caregivers of patients with anemia due to CKD (hereafter referred to as the "CKD survey"). ${ }^{25}$

Total excess costs comprised direct health care costs, transportation costs, and work productivity loss costs. Direct health care costs were reported from a payer's perspective and included costs associated with ESA administration. Transportation costs and work productivity loss costs included costs incurred by patients and caregivers separately. All costs were expressed in 2019 US dollars, consistent with the most recent complete year (ie, 2019) with the available Consumer Price Index (CPI) at the time the analyses were conducted. Direct health care costs were inflated to 2019 US dollars using the CPI for All Urban Consumers (CPI-U): Medical Care. Transportation costs and work productivity loss costs were inflated to 2019 US dollars using the CPI-U: All Items and CPI-U: Hourly Compensation, respectively.

Total excess costs were reported at the societal level annually, at the patient level annually (ie, per patient), and at the ESA administration level (ie, for each ESA administration). At the societal level, a prevalence-based approach was used to estimate annual costs of ESA administration, where costs per patient were multiplied by the number of prevalent ESA users in the US population. Annual per-patient costs of ESA administration were calculated by multiplying the total cost per administration by the annual number of administrations separately for each available frequency of administration (ie, annual frequency of 12 for once monthly, 26 for every other week, 52 for once weekly, 156 for thriceweekly administration). ${ }^{25}$
Total excess costs were reported overall and then stratified by insurance type (ie, commercial, Medicare, Medicaid, Veterans Affairs [VA], and uninsured) and administration setting (ie, in-clinic or at-home administration).

\section{POPULATION}

Data on the US adult population in 2019 were obtained from the US Census Bureau. ${ }^{26,27}$ The distribution of the US population by payer type was estimated by classifying individuals into mutually exclusive payer types using the following hierarchy: commercial (including employment-based, direct purchase, TRICARE providers, and patients with dual enrollment in commercial and Medicare or Medicaid), Medicare (including patients with dual enrollment in Medicare and Medicaid), Medicaid, VA, and uninsured. ${ }^{28}$

The number of adults with NDD-CKD receiving ESAs for the treatment of anemia in 2019 was estimated using publicly available sources that derived payer-specific estimates of the prevalence of diagnosed NDD-CKD and ESA use among patients with NDD-CKD in the United States from claims data. ${ }^{4,29}$ Due to the limited availability of prevalence estimates for certain payer types, the prevalence of diagnosed NDD-CKD among VA-insured patients was assumed to be equal to that of commercially insured patients. ${ }^{4}$ Similarly, the prevalence of ESA use among Medicaidinsured, VA-insured, and uninsured patients was assumed to be equal to that of commercially insured patients. ${ }^{29}$

The overall number of patients with NDD-CKD receiving ESAs in the United States was calculated as the sum of patients across payer types. Due to the lack of publicly available US-specific estimates, the number of patients receiving in-clinic and at-home ESA administration, as well as the frequency of in-clinic and at-home ESA administration, were estimated based on results from the CKD survey. ${ }^{25}$ To account for the fact that some population parameters were estimated prior to 2019, these parameters were adjusted for population growth based on data from the US Census Bureau. ${ }^{26,27}$

\section{DIRECT HEALTH CARE COSTS}

Direct health care costs included costs of ESA administration and monitoring costs. These costs were reported from a payer's perspective and were assumed to be the same across all payer types due to limited data on payer-specific estimates available in the literature.

Costs of ESA administration included fees such as examination fees and facility fees per ESA administration but excluded drug acquisition costs (ie, the cost per ESA unit).$^{19}$ Only patients receiving in-clinic ESAs were assumed to incur these costs. It was assumed that at-home ESA administration was conducted by patients or caregivers 


\section{FIGURE 1 Total Annual Excess Costs Associated With ESA} Administration in Patients With NDD-CKD and Anemia: Population Level ${ }^{\mathrm{a}}$

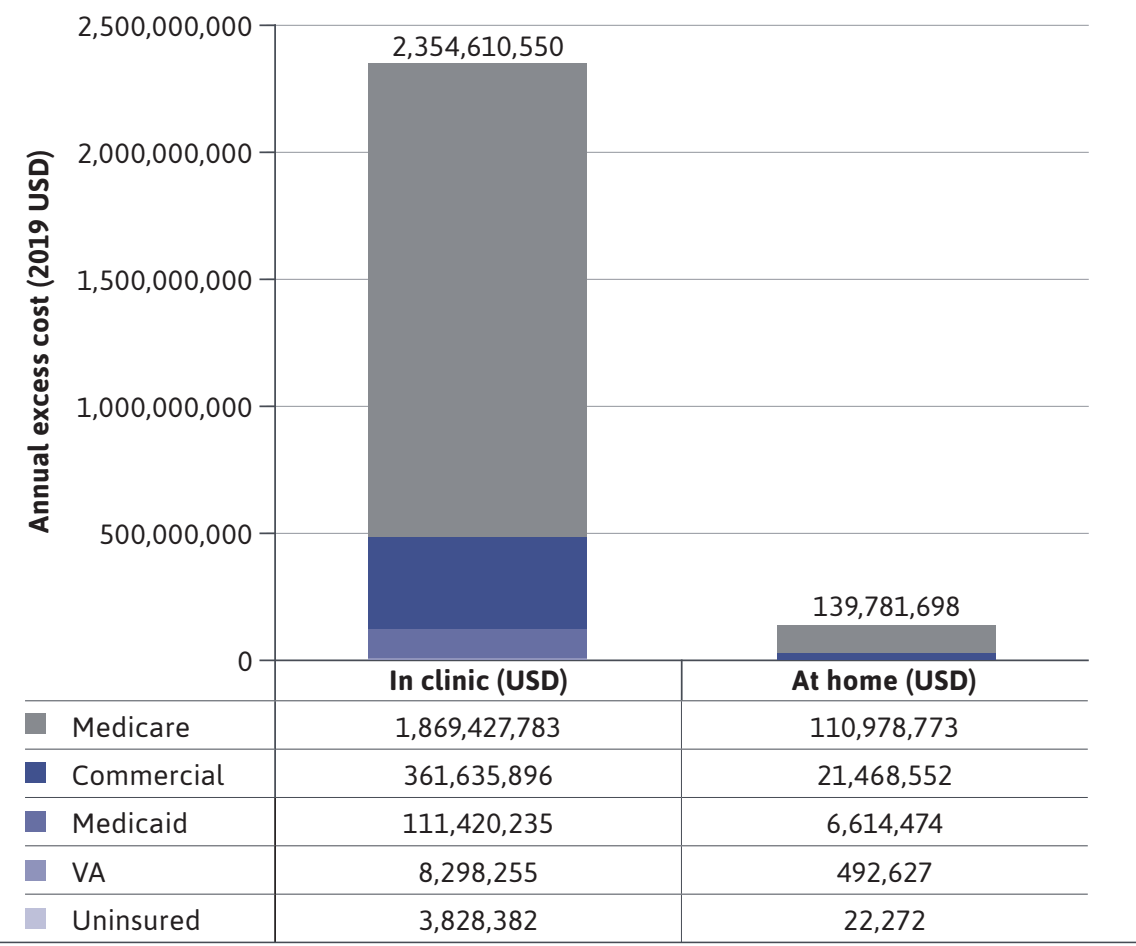

aBased on 2019 US population.

$E S A=$ erythropoiesis-stimulating agent; NDD-CKD=non-dialysis-dependent chronic kidney disease; $U S D=U S$ dollars; $V A=$ United States Department of Veterans Affairs.

directly, and therefore, was not associated with direct health care costs.

Monitoring costs included fees associated with the assessment of blood hemoglobin levels through laboratory tests and nurse consultations. ${ }^{19}$ Monitoring costs were assumed to be the same across administration settings (ie, in clinic and at home). Annual monitoring costs per patient were calculated by multiplying the cost per monitoring event by the expected annual number of monitoring events related to ESA administration. The frequency of monitoring events was estimated based on the US Food and Drug Administration recommendation to monitor patients on a monthly basis during the maintenance phase (ie, 12 monitoring events per year). ${ }^{30,31}$

Transportation Costs. Transportation costs included costs incurred for transportation to and from health care facilities to receive in-clinic ESA treatment. Per-patient costs included both patient and caregiver costs and accounted for the proportion of patients who were accompanied by a caregiver during ESA administration visits. It was assumed that no additional transportation costs were incurred for monitoring (eg, monitoring performed during ESA administration visits). The average transportation cost per administration was calculated by multiplying the average total travel time per visit, as reported by persons seeking ambulatory care in the United States (patients and caregivers separately), by the average driving speed to obtain the average distance traveled, ${ }^{32,33}$ which was then multiplied by the average cost per mile. ${ }^{34}$ Only patients receiving in-clinic ESAs were assumed to incur these costs.

Work Productivity Loss Costs. Work productivity loss costs were estimated based on a human capital approach, which assumed productivity was valued at an individual's market earnings. Per-patient costs included both patient and caregiver costs, accounting for the proportion of patients who were accompanied to visits by a caregiver.

Patients' work productivity loss costs were estimated using the average time required for ESA administration and patients' mean hourly wage. ${ }^{32}$ For in-clinic ESA recipients, the time required for ESA administration included all time spent from the moment the patient left his or her residence to the moment he or she returned (eg, including time spent traveling, waiting at the health care facility, and receiving the ESA). ${ }^{32}$ For at-home ESA recipients, the time required for ESA administration included all time spent preparing and administering ESA treatment at home. ${ }^{25}$

Caregivers' work productivity loss costs were estimated similarly, using the average time required for ESA administration from the caregivers' perspective, caregivers' mean hourly wage, and the proportion of patients with a caregiver assisting during administration. ${ }^{25,32}$

\section{SENSITIVITY ANALYSES}

Two sensitivity analyses were conducted assuming (1) monitoring requirements were equivalent for needle-based and oral formulations 


\section{FIGURE 2 Total Annual Excess Costs Associated With ESA Administration in Patients With NDD-CKD and Anemia: Breakdown by Cost Component $^{\mathrm{a}}$}

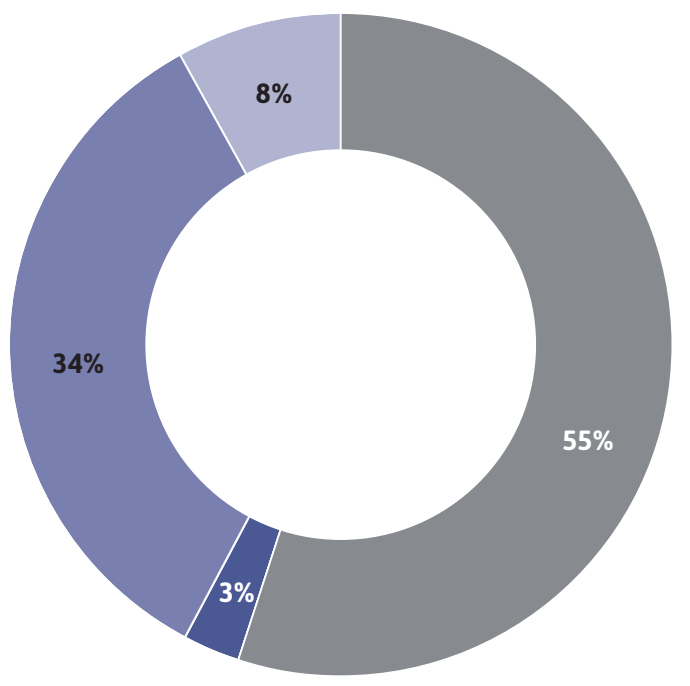

$$
\begin{aligned}
& \text { Direct health care costs } \\
& \text { Transportation costs } \\
& \text { Work productivity lost costs: patient } \\
& \text { Work productivity lost costs: caregiver }
\end{aligned}
$$

aBased on 2019 US population.

ESA = erythropoiesis-stimulating agent; NDD-CKD=non-dialysis-dependent chronic kidney disease.

(ie, \$0 excess monitoring costs) and (2) the prevalence of ESA use among uninsured individuals was $0 \%$ (ie, $\$ 0$ excess costs among uninsured patients).

\section{Results}

Annual excess costs associated with ESA administration in the United States in 2019 were estimated at \$2.5 billion, or $\$ 5,399$ per patient. In-clinic ESA administration accounted for $94.4 \%$ ( $\$ 2.4$ billion) of total annual excess costs, and at-home ESA administration accounted for the remaining 5.6\% (\$140 million; Figure 1). Medicare-insured patients accounted for $79.4 \%$ of ESA users with NDD-CKD and incurred $\$ 2.0$ billion in total annual excess costs. The remaining costs were incurred by commercially insured (\$383 million), Medicaid-insured (\$118 million), VA-insured (\$9 million), and uninsured patients (\$4 million; Figure 1). The largest contributor to total annual excess costs was direct health care costs (\$1.4 billion, 54.9\%), followed by work productivity loss costs (\$1.0 billion, $41.8 \%$ ), and transportation costs ( $\$ 81$ million, 3.3\%; Figure 2).

Total annual excess costs of in-clinic ESA administration ranged from $\$ 2,572$ per patient receiving monthly administration to $\$ 20,948$ per patient receiving thriceweekly administration, while total annual excess costs of at-home ESA administration ranged from $\$ 1,123$ for monthly administration to $\$ 2,109$ for thrice-weekly administration (Figure 3). At the ESA administration level, total excess costs were estimated at \$128 per in-clinic ESA administration and \$7 per at-home ESA administration, excluding monitoring costs (Supplementary Table 1, available in online article).

\section{POPULATION}

The number of adults with diagnosed NDD-CKD in the United States in 2019 was estimated to be 8,916,742 based on an overall prevalence of $3.6 \%$ (Table 1). This prevalence was derived from payer-specific estimates of diagnosed NDD-CKD for commercially insured $(1.3 \%$; also used for VA-insured patients), ${ }^{35}$ Medicare-insured (22.0\%), ${ }^{35}$ Medicaid-insured (3.5\%), ${ }^{35}$ and uninsured patients (0.1\%). ${ }^{5}$

Among patients with NDD-CKD, it was estimated that 462,005 received ESAs for the treatment of anemia, based on an overall prevalence of ESA use of 5.2\% in the NDD-CKD population (Table 1). This prevalence was similarly derived from payer-specific estimates of ESA use among commercially insured patients (3.0\%; also used for Medicaid-insured, VA-insured, and uninsured patients) and Medicare-insured patients (6.4\%). ${ }^{29}$

Based on the results of the CKD survey, the numbers of in-clinic and at-home ESA recipients were estimated to be 367,893 and 94,112, respectively (Figure 4). Among in-clinic recipients, the most common dosing frequency was once monthly (34.3\%), while once every other week was the most common dosing frequency among at-home recipients (45.5\%; Figure 4).

\section{DIRECT HEALTH CARE COSTS}

Annual excess direct health care costs associated with ESA administration (ie, costs of ESA administration and monitoring costs) were estimated at $\$ 1.4$ billion, or $\$ 2,965$ per patient. Annual direct health care costs associated with in-clinic ESA administration ranged from $\$ 1,731$ per patient receiving monthly administration to $\$ 10,018$ per patient receiving thrice-weekly administration. Annual per-patient direct health care costs associated with at-home ESA administration were $\$ 1,041$, regardless of dosing frequency.

Direct costs per in-clinic ESA administration (ie, excluding monitoring costs) were estimated at $\$ 58$ (Supplementary Table 1). $\cdot{ }^{19}$ Annual excess costs of in-clinic ESA administration 


\section{FIGURE 3 Annual Excess Costs Associated With ESA Administration in Patients With NDD-CKD and Anemia: Breakdown by Setting of Administration and Dosing Frequency $\mathrm{a}, \mathrm{b}, \mathrm{c}$}

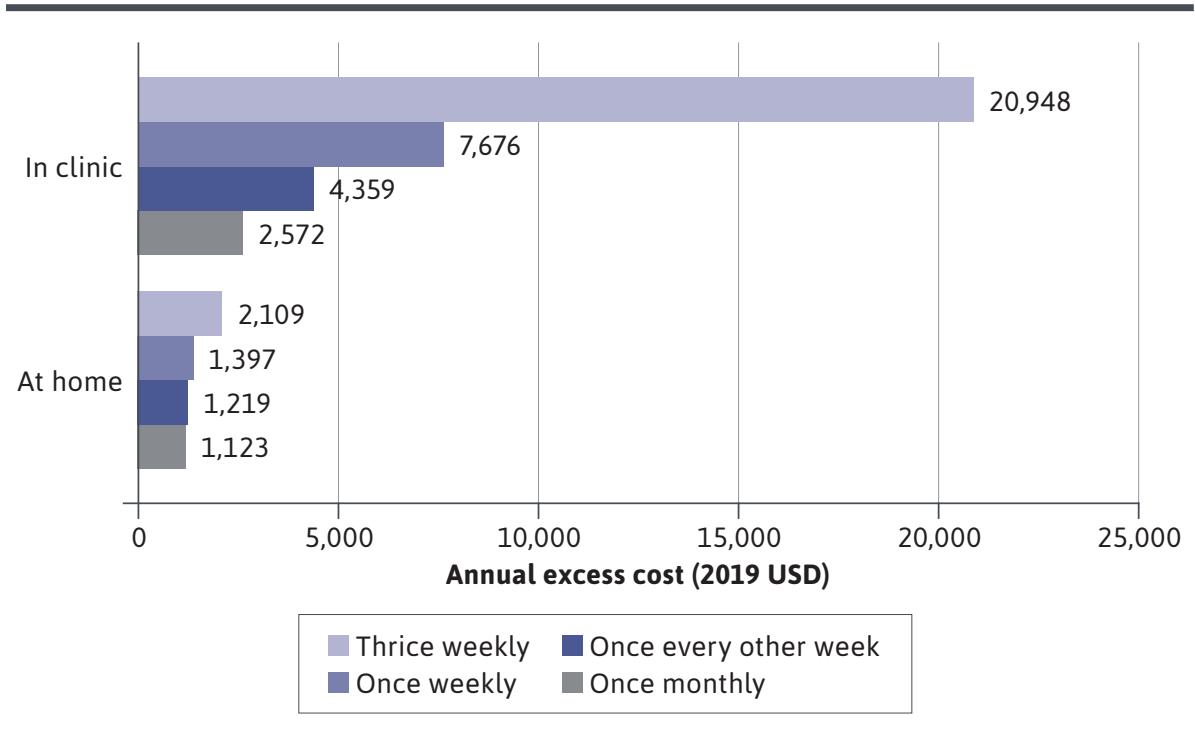

aThe annual frequency of ESA administration was assumed to be 12 for patients receiving ESA administration 1 time/month, 26 for patients receiving ESA administration once every other week, 52 for patients receiving ESA administration 1 time/week, and 156 for patients receiving ESA administration 3 times/week.

${ }^{b}$ Based on 2019 US population.

'Annual excess costs are per patient.

$E S A=$ erythropoiesis-stimulating agent; NDD-CKD=non-dialysis-dependent chronic kidney disease; USD $=$ US dollars. ranged from $\$ 691$ per patient receiving monthly administration to $\$ 8,978$ per patient receiving thrice-weekly administration. Direct costs of athome ESA administration (ie, excluding monitoring costs) were assumed to be \$0 (Supplementary Table 1).

Direct health care costs associated with each monitoring visit were estimated at $\$ 87$ (Supplementary Table 1), corresponding to an annual cost of \$1,041, assuming monthly monitoring visits. Costs per visit included those associated with hemoglobin tests (\$3), the cost of a blood draw (\$3), and the cost of a nurse consultation $(\$ 80) \cdot{ }^{19}$

Transportation Costs. Annual excess transportation costs associated with ESA administration were estimated at
\$81 million, or \$176 per patient. At the patient level, annual transportation costs associated with in-clinic ESA administration (including both patient and caregiver costs) ranged from $\$ 63$ for monthly administration to $\$ 822$ for thrice-weekly administration. At-home administration was assumed to incur $\$ 0$ in transportation costs.

At the ESA administration level, total transportation costs (including both patient and caregiver costs) associated with in-clinic ESA administration were estimated at \$5 (Supplementary Table 1). From the patient perspective, each in-clinic administration visit incurred \$4 of excess transportation costs. This cost was estimated based on an average self-reported total travel time of 33 minutes for an in-clinic appointment, ${ }^{32}$ an average driving speed of $37.1 \mathrm{mph},{ }^{33}$ and an average cost per mile of 20 cents. ${ }^{34}$ From the caregiver perspective, each in-clinic administration visit incurred an average excess transportation cost of \$1. This cost was estimated based on an average self-reported total travel time of 48 minutes for an in-clinic appointment, ${ }^{32}$ an average driving speed of $37.1 \mathrm{mph},{ }^{33}$ an average cost per mile of 20 cents, ${ }^{34}$ and an assumption that $20 \%$ of patients were accompanied to visits by a caregiver. ${ }^{32}$

Work Productivity Loss Costs. Annual excess work productivity loss costs associated with ESA administration were estimated at $\$ 1.0$ billion, or $\$ 2,257$ per patient. These costs included $\$ 846$ million in patient work productivity loss costs and \$197 million in caregiver work productivity loss costs. Annual work productivity loss costs associated with in-clinic ESA administration (including both patient and caregiver costs) ranged from $\$ 777$ per patient receiving monthly administration to $\$ 10,107$ per patient receiving thrice-weekly administration; for athome administration, these costs ranged from $\$ 82$ per patient receiving monthly administration to $\$ 1,069$ per patient receiving thrice-weekly administration.

For patients, excess work productivity loss costs were estimated at \$53 per in-clinic ESA administration (Supplementary Table 1). This cost was calculated using an average hourly wage of $\$ 28$ (based on the characteristics of an average patient) and an average time of 115 minutes to seek care. ${ }^{32}$ At-home ESA administration was estimated to incur excess work productivity loss costs of \$5 per administration (Supplementary Table 1) based on an average hourly wage of \$28 and an average duration of ESA administration of 10 minutes. ${ }^{25,32}$ 


\section{TABLE 1 Population and Prevalence of ESA Use Among Adults With NDD-CKD in the United States in 2019}

\begin{tabular}{|c|c|c|c|c|c|c|}
\hline Component & Overall & Commercial & Medicare & Medicaid & VA & Uninsured \\
\hline US adult population ${ }^{a}$ (aged $\geq 18$ years) & $251,116,064$ & $175,230,701$ & $26,205,859$ & $20,677,666$ & $4,020,920$ & $24,980,917$ \\
\hline Prevalence of NDD-CKD diagnosis, \% & 3.6 & $1.3^{\mathrm{b}}$ & $22.0^{\mathrm{b}}$ & $3.5^{b}$ & $1.3^{\mathrm{b}}$ & $0.1^{\mathrm{b}}$ \\
\hline Number of adults with NDD-CKD & $8,916,742$ & $2,348,968$ & $5,765,289$ & 723,718 & 53,900 & 24,867 \\
\hline $\begin{array}{l}\text { Prevalence of ESA use among adults } \\
\text { with NDD-CKD, \% }\end{array}$ & 5.2 & $3.0^{c}$ & $6.4^{c}$ & $3.0^{c}$ & $3.0^{c}$ & $3.0^{c}$ \\
\hline $\begin{array}{l}\text { Number of adults with NDD-CKD } \\
\text { receiving ESAs }\end{array}$ & 462,005 & 70,958 & 366,806 & 21,862 & 1,628 & 751 \\
\hline
\end{tabular}

aPopulation estimates are based on the 2019 civilian non-institutionalized population. The proportion of adults in this population was assumed to be the same as the proportion of adults in the 2019 resident population. ${ }^{26,27}$

bThe prevalence of NDD-CKD in the commercial, Medicare, and Medicaid populations was reported based on estimates published by the Centers for Disease

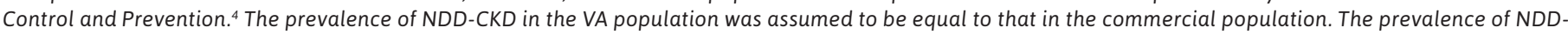
CKD in the uninsured population was derived using Medical Expenditure Panel Survey Household Component data. ${ }^{5}$

The prevalence of ESA use in the commercial and Medicare NDD-CKD populations was reported based on estimates obtained using IBM MarketScan and CMS

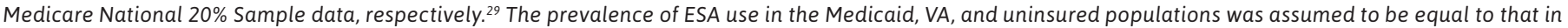
the commercial population.

CMS=Centers for Medicare \& Medicaid Services; ESA = erythropoiesis-stimulating agent; NDD-CKD=non-dialysis-dependent chronic kidney disease; VA=US Department of Veterans Affairs.

For caregivers, excess work productivity loss costs were estimated at $\$ 12$ per in-clinic ESA administration (Supplementary Table 1). This cost was calculated using an average hourly wage of $\$ 26$ (based on the characteristics of an average caregiver), an average of 139 minutes spent accompanying patients to receive care, with the assumption that $20 \%$ of patients are accompanied by a caregiver. ${ }^{32}$ Each at-home ESA administration was estimated to incur excess work productivity loss costs of \$2 per administration. This cost was calculated using an average hourly wage of \$26 and an average duration of ESA administration of 10 minutes,$^{32}$ with the assumption that $53 \%$ of patients are assisted by a caregiver for at-home administration..$^{25}$

\section{SENSITIVITY ANALYSES}

Monitoring Costs. Assuming monitoring requirements were equivalent for needle-based and oral formulations (ie, \$0 excess monitoring costs), total annual excess costs associated with ESA administration in the United States in 2019 were estimated at $\$ 2.0$ billion, or $\$ 4,358$ per patient, which represents a $\$ 0.5$ billion (19\%) reduction compared with the base-case scenario (Supplementary Figure 1, available in online article).

Prevalence of ESA Use Among Uninsured Patients. Assuming the prevalence of ESA use among uninsured individuals was $0 \%$ (ie, \$0 excess costs among uninsured patients), the total estimated number of adults receiving ESAs in the United States was 461,254, which represents a reduction of $751(0.2 \%)$ patients receiving ESAs. Total annual excess costs were comparable to the base-case scenario (\$2.5 billion).

\section{Discussion}

The findings of this study suggest that the needle-based administration of ESAs is associated with a considerable economic burden, which was estimated at $\$ 2.5$ billion in the United States in 2019. More than 90\% of this excess cost was due to in-clinic ESA administration, suggesting that resources associated with ESA administration visits account for the vast majority of costs that could be avoided with the introduction of novel oral medications. In addition, direct health care costs and patients' work productivity loss costs, particularly those resulting from in-clinic ESA administration, were the 2 main drivers of total excess costs, respectively accounting for $54.9 \%$ and $33.9 \%$ of total excess costs. Further, in both administration settings, Medicareinsured patients incurred the majority of costs due to the high prevalence of NDD-CKD and ESA use in this population, which resulted in nearly $80 \%$ of total excess costs incurred by Medicare-insured patients.

To the best of our knowledge, this study is the first to provide a societal perspective on ESA administration costs that could potentially be avoided with the introduction of a new oral treatment for anemia due to CKD with comparable safety and efficacy to ESAs. Most prior studies that 


\section{FIGURE 4 Frequency of ESA Administration in Patients With NDD-CKD by Setting ${ }^{\mathrm{a}, \mathrm{b}}$}

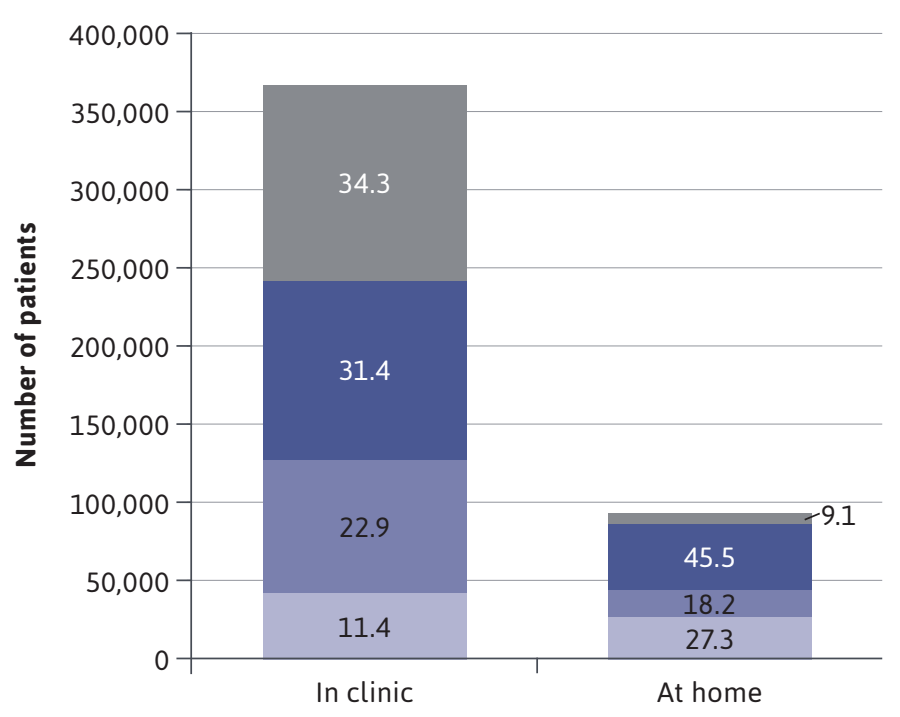

ESA administration frequency, \%

Thrice weekly ance every other week Once weekly Once monthly

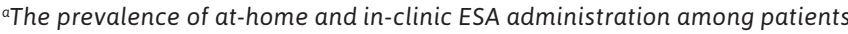
with anemia and CKD was estimated using the results of a survey conducted in parallel with the current study. 25 Results were provided from 54 respondents with anemia and CKD (NDD or DD) who reported currently receiving ESA treatment to manage their anemia. Of note, 8 out of 43 respondents receiving ESAs in clinic had an unknown frequency of ESA administration and were excluded from the analyses reported above. Results were largely consistent across NDD-CKD and DD-CKD subgroups.

bBased on 2019 US population.

$D D=$ dialysis-dependent; $E S A$ = erythropoiesis-stimulating agent; NDD-CKD =non-dialysis-dependent chronic kidney disease.

assessed the economic burden associated with ESAs and their administration did not focus on patients with NDDCKD, who may incur the greatest excess costs associated with ESA administration. ${ }^{36-39}$

In addition, studies that included patients with NDDCKD were conducted more than a decade ago and thus may not provide reliable contemporary estimates due to changes in ESA reimbursement policies and prescribing labels over the past decade. ${ }^{17,23,24,40-42}$ Further, most of these studies exclusively assessed the burden associated with ESAs from the patient perspective or only considered direct health care costs, ${ }^{17,23,24}$ which does not allow for a comprehensive assessment of excess costs associated with the needle-based administration of ESAs.
The results of the current study suggest that the needlebased administration of ESAs may account for a notable proportion of the economic burden of ESA-treated patients. A 2007 study by Maddux et al reported that ESA recipients with NDD-CKD incurred total direct health care costs of $\$ 3,876$ per month (ie, annual costs of $\$ 46,512){ }^{43}$ an estimate that included costs incurred by patients, payers, and third parties. The present estimate of $\$ 2,965$ per year in direct health care costs incurred exclusively by payers due to the needle-based route of administration of ESAs and associated monitoring requirements may account for an important proportion of total health care costs among ESA-treated patients with NDD-CKD.

The needle-based route of ESA administration may also have an impact on patient and caregiver HRQOL, which was not measured as part of this study. For example, patients' fear of needles and potential injection-site reactions (eg, skin rash) may impact HRQOL and further compound the overall burden of ESA administration. ${ }^{31}$ Furthermore, while the current study assessed the economic impact of time spent receiving therapy, the intrinsic value of an individual's time spent receiving therapy also warrants consideration. Clinic visits, transportation time, and work loss may result in diminished quality of life and add to the already high burden of anemia due to CKD. Therefore, an oral treatment merits consideration as a convenient alternative to ESAs for the treatment of anemia due to CKD.

Despite the cost and burden associated with ESA administration, current treatment options for anemia in patients with CKD requiring ESAs remain limited to needle-based medications. In a recent survey, nearly $50 \%$ of patients and caregivers of patients with anemia and NDD-CKD expressed a preference for an oral anemia treatment. ${ }^{25}$ Therefore, the use of an oral treatment with a safety and efficacy profile comparable to that of ESAs has the potential to improve patients' satisfaction with treatment in addition to avoiding costs associated with ESA administration.

HIF-PHIs have been shown to improve anemia by stimulating endogenous erythropoietin production and restoring iron metabolism in ongoing clinical trials. ${ }^{44}$ Although comparative data are currently limited, it is thought that this novel mechanism will enable a safer and more effective correction of anemia compared with ESAs. ${ }^{45}$ Once additional clinical data are available, further studies will be warranted to account for potential differences in the safety and efficacy of HIF-PHIs compared with ESAs and the potential cost implications of these differences.

\section{LIMITATIONS}

The present study is subject to some limitations. For certain payer types, the prevalence of NDD-CKD and ESA use was 
assumed to be equal to that of commercially insured patients due to a lack of published payer-specific estimates. This assumption may have underestimated the societal cost associated with ESA administration, as estimates for the commercially insured population were the lowest estimates among available payer types.

Data on the setting (ie, in clinic vs at home) and frequency of ESA administration were derived from a recent survey in which the sample may not be representative of the general US population. Moreover, there was a scarcity of information on certain cost components (ie, transportation costs and work productivity loss costs) specific to the ESA-treated NDD-CKD population. Hence, values derived from the general population receiving ambulatory care were used for these components. However, values in the general population were very similar to CKD-specific estimates found in the CKD survey. For example, $20 \%$ of adults in the US general population were reportedly accompanied to ambulatory visits by a caregiver compared with $23.7 \%$ of patients accompanied to ESA administration visits as reported in the CKD survey. ${ }^{25,32}$

Because of the scarcity of publicly available estimates of comparable direct health care costs for all payer types, direct health care costs of Medicare-insured patients were applied to individuals with other types of insurance coverage; this extrapolation may have underestimated health care costs, especially among commercially insured individuals who are known to incur higher costs than Medicare beneficiaries. ${ }^{46-48}$

Although this analysis adopted a societal perspective, which encompassed costs beyond direct health care costs, this study was limited to specific costs for which estimates were available in the literature.
Accordingly, total excess costs may be underestimated due to additional factors related to the needle-based administration of ESAs (eg, record keeping costs, drug ordering, injection-site reactions, biohazard waste management, disposal of unused medication, and training costs for at-home administration) or potential consequences of increased caregiver burden (eg, depression, sleep disorders), which were not captured in this study due to a lack of cost estimates in the literature.

Finally, this study focused on the NDD-CKD population, and costs may not be generalizable to the $\mathrm{DD}-\mathrm{CKD}$ population.

\section{Conclusions}

The findings of this study suggest that needle-based administration of ESAs in patients with NDD-CKD may be associated with a substantial economic burden. If all ESA-treated patients with NDD-CKD and anemia were to receive an oral treatment with comparable safety and efficacy as when receiving ESAs, potential savings associated with oral administration would amount to approximately $\$ 2.5$ billion from a societal perspective. As new data become available, further studies will be warranted to assess excess costs associated with ESA administration, taking into account potential differences between new oral treatments and ESAs in terms of safety and efficacy.

\section{DISCLOSURES}

This study was funded by Otsuka Pharmaceutical Development \& Commercialization, Inc., and Akebia Therapeutics, Inc. The study sponsors participated in the study design, data collection, analysis, interpretation of the data, writing of the report, and in the decision to submit the manuscript for publication.
Gauthier-Loiselle, Cloutier, Serra, Bungay, and Guérin are employees of Analysis Group, Inc., a consulting firm that received funding from Otsuka Pharmaceutical Development \& Commercialization, Inc., for the conduct of this study. Michalopoulos was an employee of Otsuka Pharmaceutical Development \& Commercialization, Inc., at the time the study was conducted. Szabo is an employee of Akebia Therapeutics, Inc.

\section{ACKNOWLEDGMENTS}

Medical writing assistance was provided by Samuel Rochette, an employee of Analysis Group, Inc., with funding for this assistance provided by Otsuka Pharmaceutical Development \& Commercialization, Inc., and Akebia Therapeutics, Inc.

\section{REFERENCES}

1. Kidney Disease: Improving Global Outcomes (KDIGO) CKD Work Group. KDIGO 2012 clinical practice guideline for the evaluation and management of chronic kidney disease. Kidney Int Suppl. 2013;3(1):1-150.

2. Romagnani P, Remuzzi G, Glassock R, et al. Chronic kidney disease. Nat Rev Dis Primers. 2017;3:17088.

3. Thomas MC, Brownlee M, Susztak K, et al. Diabetic kidney disease. Nat Rev Dis Primers. 2015;1:15018.

4. Centers for Disease Control and Prevention. Prevalence of CKD in the health care system population: percentage of patients with ICD-9-CM or ICD-10-CM codes indicating CKD or ESRD. Accessed August 5, 2020. https://nccd.cdc.gov/ckd/detail. aspx?Qnum=Q79\#refreshPosition

5. Ozieh MN, Bishu KG, Dismuke CE, Egede LE. Trends in healthcare expenditure in United States adults with chronic kidney disease: 2002-2011. BMC Health Serv Res. 2017;17(1):368.

6. United States Renal Data System. US Renal Data System 2019 annual data report: epidemiology of kidney disease in the United States. 2019. Accessed August 3, 2020. https://www.usrds.org/2019/ view/USRDS_2019_ES_final.pdf 
7. United States Renal Data System. Chapter 7: healthcare expenditures for persons with CKD. 2018. Accessed August 3, 2020. https://www.usrds.org/2018/ view/v1_07.aspx

8. Golestaneh L, Alvarez PJ, Reaven NL, et al. All-cause costs increase exponentially with increased chronic kidney disease stage. Am J Manag Care. 2017;23(10 suppl):S163-72.

9. Mix TCH, St peter WL, Ebben J, et al. Hospitalization during advancing chronic kidney disease. Am J Kidney Dis. 2003;42(5):972-81.

10. Stauffer ME, Fan T. Prevalence of anemia in chronic kidney disease in the United States. PLoS One. 2014;9(1):e84943.

11. Eriksson D, Goldsmith D, Teitsson S, Jackson J, van Nooten F. Cross-sectional survey in CKD patients across Europe describing the association between quality of life and anaemia. BMC Nephrol. 2016;17(1):97.

12. Pergola PE, Pecoits-Filho R, Winkelmayer WC, et al. Economic burden and health-related quality of life associated with current treatments for anaemia in patients with CKD not on dialysis: a systematic review. Pharmacoecon Open. 2019;3(4):463-78.

13. Spinowitz B, Pecoits-Filho R, Winkelmayer WC, et al. Economic and quality of life burden of anemia on patients with CKD on dialysis: a systematic review. J Med Econ. 2019;22(6):593-604.

14. Kidney Disease: Improving Global Outcomes (KDIGO) Anemia Work Group. KDIGO clinical practice guideline for anemia in chronic kidney disease. Kidney Int Suppl. 2012;2(4):279-335.

15. Rottembourg J, Tilleul P, Deray G, et al. Cost of managing anemia in endstage renal disease: the experience of five French dialysis centers. Eur J Health Econ. 2015;16(4):357-64.
16. Saueressig U, Kwan JT, De Cock E, Sapede C. Healthcare resource utilization for anemia management: current practice with erythropoiesis-stimulating agents and the impact of converting to once-monthly C.E.R.A. Blood Purif. 2008;26(6):537-46.

17. Globe D, Glidden D, Hertel J, et al. Assessment of the impact of weekly versus monthly erythropoiesis stimulating protein therapy on patients with CKD and their families. Nephrol Nurs J. 2007;34(2):151-61.

18. Hayat A, Haria D, Salifu MO. Erythropoietin stimulating agents in the management of anemia of chronic kidney disease. Patient Prefer Adherence. 2008;2:195-200.

19. Dahl NV, Kaper RF, Strauss WE, Corvino FA, Zivkovic M. Cost-effectiveness analysis of intravenous ferumoxytol for the treatment of iron deficiency anemia in adult patients with non-dialysis-dependent chronic kidney disease in the USA. Clinicoecon Outcomes Res. 2017;9:557-67.

20. Lavelle T, Michalopoulos S, Margaretos N, et al. A review of costeffectiveness analyses for anemia in chronic kidney disease. Paper presented at: National Kidney Foundation 2019 Spring Clinical Meeting; May 2019; Boston, MA. Accessed November 3, 2021. https://www.ajkd.org/issue/S02726386(18)X0007-9\#NationalKidneyFoundat ion2019SpringClinicalMeetingsAbstracts

21. Chen N, Hao C, Peng X, et al.

Roxadustat for anemia in patients with kidney disease not receiving dialysis. $\mathrm{N}$ Engl J Med. 2019;381(11):1001-10.

22. Martin ER, Smith MT, Maroni BJ, Zuraw QC, deGoma EM. Clinical trial of vadadustat in patients with anemia secondary to stage 3 or 4 chronic kidney disease. Am J Nephrol. 2017;45(5):380-88.

23. Akers GR, Bucaloiu ID, Bermudez MC, et al. A comparison of home versus clinic administration of epoetin alfa in chronic kidney disease patients. J Clin Outcomes Manag. 2010;17(2):62-6.
24. Bernardo M, Crawford P, Hertel J, et al. Assessment of time and practice resources required to provide weekly or monthly erythropoiesis-stimulating protein therapy to chronic kidney disease patients in the physician office setting. J Manag Care Pharm. 2006;12(9):714-25. doi: 10.18553/jmcp.2006.12.9.714

25. Michalopoulos E, Gauthier-Loiselle M, Sanon M, et al. Reported caregiver burden in chronic kidney disease with and without anemia: a US-based survey. Abstract \#PO0272. Presented at: American Society of Nephrology; October 22, 2020; virtual event. Accessed October 28, 2021. https://www.asn-online.org/education/ kidneyweek/2020/program-abstract. aspx?controlId $=3439120$

26. US Census Bureau. Monthly population estimates for the United States: April 1, 2010 to December 1, 2020. 2019. Accessed January 31, 2020. https://www. census.gov/data/tables/time-series/ demo/popest/2010s-national-total.html

27. US Census Bureau. Estimates of the total resident population and resident population age 18 years and older for the United States and Puerto Rico: July 1, 2019. 2019. Accessed July 1, 2019. https://www.census.gov/data/tables/ time-series/demo/popest/2010snational-total.html

28. US Census Bureau. American community survey tables for health insurance coverage. 2018. 2018;HI-05. Accessed January 31, 2020. https://www.census. gov/data/tables/time-series/demo/ health-insurance/acs-hi.2018.html

29. St Peter WL, Guo H, Kabadi S, et al. Prevalence, treatment patterns, and healthcare resource utilization in Medicare and commercially insured nondialysis-dependent chronic kidney disease patients with and without anemia in the United States. BMC Nephrol. 2018;19(1):67.

30. Aranesp. Prescribing information. Amgen, Inc; 2019. Accessed March 3, 2020. https://www.accessdata.fda.gov/drugsatfda_docs/label/2019/103951s5378lbl. pdf 
31. Procrit. Prescribing information. Amgen, Inc; 2018. Accessed March 3, 2020. https://www.janssenlabels.com/ package-insert/product-monograph/ prescribing-information/PROCRIT-pi.pdf

32. Ray KN, Chari AV, Engberg J, Bertolet M, Mehrotra A. Opportunity costs of ambulatory medical care in the United States. Am J Manag Care. 2015;21(8):567-74.

33. American Automobile Association. American driving survey, 2014-2017. February 2019. Accessed February 21, 2020. https://aaafoundation.org/ wp-content/uploads/2019/02/2019 AAAFTS-ADS-Brief.pdf

34. Internal Revenue Service. Standard mileage rates. 2019. Accessed February 21, 2020. https://www.irs.gov/ tax-professionals/standard-mileage-rates

35. Centers for Disease Control and Prevention. Chronic kidney disease (CKD) surveillance system. 2016. Accessed December 20, 2019. https://nccd.cdc.gov/ CKD/detail.aspx?Qnum $=$ Q10

36. Churchill DN, Macarios D, Attard C, Kallich J, Goeree R. Costs associated with erythropoiesis-stimulating agent administration to hemodialysis patients. Nephron Clin Pract. 2007;106(4):c193-8.

37. Courtney AE, McNamee PT, Maxwell AP. Cost should be the principal determinant of choice of erythropoiesis-stimulating agent in chronic haemodialysis patients. Nephron Clin Pract. 2007;107(1):c14-19.
38. Stephens JM, Emerson LC, Spry LA, et al. Time savings of weekly versus three-times-per-week administration of erythropoiesis stimulating agents in United States dialysis patients. Curr Med Res Opin. 2016;32(2):313-20.

39. Woodland AL, Murphy SW, Curtis BM, Barrett BJ. Costs associated with intravenous darbepoetin versus epoetin therapy in hemodialysis patients: a randomized controlled trial. Can J Kidney Health Dis. 2017;4:2054358117716461.

40. Coritsidis GN, Maglinte GA, Acharya A, et al. Anemia management trends in hospital-based dialysis centers (HBDCs), 2010 to 2013. Clin Ther. 2014;36(3):408-18.

41. Thamer M, Zhang Y, Kshirsagar O, Cotter DJ, Kaufman JS. Erythropoiesisstimulating agent use among non-dialysis-dependent CKD patients before and after the trial to reduce cardiovascular events with aranesp therapy (TREAT) using a large US health plan database. Am J Kidney Dis. 2014;64(5):706-13.

42. Wang C, Kane R, Levenson M, et al. Association between changes in CMS reimbursement policy and drug labels for erythrocyte-stimulating agents with outcomes for older patients undergoing hemodialysis covered by feefor-service Medicare. JAMA Intern Med. 2016;176(12):1818-25.
43. Maddux FW, Shetty S, del Aguila MA, Nelson MA, Murray BM. Effect of erythropoiesis-stimulating agents on healthcare utilization, costs, and outcomes in chronic kidney disease. Ann Pharmacother. 2007;41(11):1761-69.

44. Maxwell PH, Eckardt KU. HIF prolyl hydroxylase inhibitors for the treatment of renal anaemia and beyond. Nat Rev Nephrol. 2016;12(3):157-68.

45. Locatelli F, Del Vecchio L. Are we approaching a new era in the treatment of anemia of chronic kidney disease patients? Ann Transl Med. 2019;7(suppl 8):S333.

46. White C, Whaley CM. Prices paid to hospitals by private health plans are high relative to Medicare and vary widely. Findings from an employer-led transparency initiative. RAND Corporation. 2019. Accessed January 13, 2021. https:// www.rand.org/pubs/research_reports/ $\underline{\text { RR3033.html }}$

47. Pelech DM. Prices for physicians' services in Medicare Advantage and commercial plans. Med Care Res Rev. 2020;77(3):236-48.

48. Pelech D. An analysis of privatesector prices for physicians' services. January 2018. Accessed January 13, 2021. https://www.cbo.gov/system/ files/115th-congress-2017-2018/ workingpaper/53441-workingpaper.pdf 